\title{
El esfuerzo público de desfamilización. Propuesta de medición y análisis descriptivo para la Unión Europea (1970-1999)
}

\author{
María Jesús Rodríguez García \\ Clemente J. Navarro Yáñez
}

Universidad Pablo de Olavide. Centro de Sociología y Políticas Locales

mjrodgar@upo.es

cnavyan@upo.es

\section{Resumen}

Este artículo presenta una propuesta metodológica para medir el esfuerzo público de desfamilización en quince países europeos. Junto a indicadores clásicos (como el gasto familiar o la escolarización de los menores), se incluyen medidas de conciliación entre vida doméstica y laboral para conocer la acción pública en relación con este nuevo riesgo familiar. Una vez validados los indicadores mediante análisis factorial, se utilizan para realizar un análisis descriptivo. Los resultados muestran la utilidad y la validez de los indicadores propuestos para realizar comparaciones entre países y/o en diferentes momentos del tiempo, así como la importancia del indicador de conciliación para conocer tanto la intensidad como las estrategias del esfuerzo público.

Palabras clave: desfamilización, riesgos sociales, estado del bienestar, análisis comparado, análisis longitudinal, análisis entre países, medición.

\begin{abstract}
This article presents a proposal to measure public effort of de-familization in 15 european countries. Together with others classical measures (public expenditure in family and child educational services) measures of conciliation between labour and family life is included as a dimension of de-familization in order to consider public actions related to new family risks. After a factorial analysis to validate the proposal, a comparative analysis is developed. The results seem to validate the proposal measurement and the importance of the conciliation index to capture the intensity of public effort as well as different strategies from cross-national and longitudinal perspectives.
\end{abstract}

Key words: de-familization process, social risks, welfare state, comparative analysis, longitudinal analysis, cross-national analysis, measurement

\section{Sumario}

1. Familia y estado del bienestar en las sociedades postindustriales: sobre nuevos riesgos sociales y desfamilización

2. El esfuerzo estatal de «desfamilización»: dimensiones e indicadores básicos para el análisis comparado
3. El esfuerzo público de desfamilización en la Unión Europea (1970-1999): dimensionalidad, cambio en el tiempo y diferencias entre grupos de países

4. A modo de conclusiones

Referencias bibliográficas 
Los cambios que vienen produciéndose en la familia, y en particular respecto a la incorporación femenina al mercado de trabajo, están dando lugar a un renovado debate en torno a la primera y, con ello, al papel del estado como provisor de los servicios de cuidado que anteriormente se producían en ella a través de las responsabilidades asumidas por la mujer en el ámbito privado. En buena medida, este asunto viene analizándose a través del concepto de desfamilización, entendido como conjunto de oportunidades existentes para que las familias puedan aliviar la absorción de riesgos sociales, que, por otro lado, se han diversificado, lo que ha hecho necesaria la creación de fórmulas alternativas o complementarias a la autoprovisión de servicios por parte de la familia.

Este artículo se centra en hacer una propuesta para la medición del esfuerzo de desfamilización que realizan los estados del bienestar, considerando para ello las medidas que desarrollan para procurar el bienestar de la familia en general, así como la atención de los miembros dependientes (menores y mayores) y la posibilidad de conciliación entre vida doméstica y vida laboral.

Después de plantear, en el primer apartado, el marco en el que se sitúa el concepto de desfamilización y su alcance analítico, en el segundo, se da cuenta del proceso de operacionalización desarrollado para elaborar un indicador de esfuerzo público de desfamilización, así como de sus tres dimensiones principales (gasto en familia, atención a dependientes y conciliación). En el tercer apartado, se presentan algunas pruebas sobre su validez para realizar análisis comparados a través de su aplicación a quince países de la Unión Europea durante el periodo 1970-1999. Estas pruebas vienen a confirmar las dimensiones establecidas y a mostrar la importancia de incluir la conciliación como un aspecto básico del esfuerzo público de desfamilización, tal y como se señala en el último apartado de conclusiones.

\section{Familia y estado del bienestar en las sociedades postindustriales: sobre nuevos riesgos sociales y desfamilización}

En términos generales, el estudio de la familia ha venido mostrando ciertos cambios en su tamaño y estructura, así como en las orientaciones y los valores acerca de ella como unidad de convivencia, que apuntan a dinámicas sociales más amplias. Ambas cuestiones se vienen reflejando en términos de caída de la fecundidad, aumento de la tasas de divorcio, reconocimiento de parejas de hecho o, en general, nuevas formas de hogares. También en los nuevos roles sociales de la mujer, las orientaciones hacia el trabajo doméstico, las actitudes y la incorporación efectiva de ésta al mercado laboral o las nuevas pautas en la división de las tareas domésticas. Aspectos, todos ellos, que vienen mostrando que la familia, aun conservando su papel como institución social bási$\mathrm{ca}$, ha cambiado su morfología y su dinámica internas en el contexto de las sociedades postindustriales (Semyonov, 1980; Ferrera, 1993; Clark, 1998; Esping-Andersen, 1999a).

Ahora bien, la cuestión es que tales cambios han provocado, asimismo, nuevas formas de relación entre la familia y el estado o, si se prefiere, nuevas for- 
mas de intervención estatal sobre la familia. Así, los nuevos roles de la mujer en la esfera pública y, en particular, su incorporación al mercado laboral, están dando lugar a un cambio progresivo en la forma modal de convivencia, desde el modelo de familia sustentada por un trabajador, el varón principalmente (male-bread winner), a otro en el que la mujer participa en tal sustento (dual earner family). Cuestión que se relaciona con las necesidades de cuidado de la población dependiente (infancia y mayores). Cuando menos, se trata de una situación que obliga a la familia — a sus miembros - a buscar alternativas a la provisión doméstica y femenina de servicios de ciudado, entre las que se puede contar la acción estatal.

Más en general, lo anterior viene a significar que, junto a los tradicionales riesgos sociales que dieron sentido al surgimiento y a la extensión del estado del bienestar, las sociedades postindustriales dan lugar a nuevos riesgos en relación con esta "nueva familia» (Ferrara, 1993; Scheiwe, 1997). En concreto, los riesgos tradicionales se referían principalmente a la integración sociolaboral de los trabajadores, que, en relación con la familia, significaba, fundamentalmente, considerar el coste que para éstos tenían las cargas familiares (Castel, 1991). En este caso, la intervención estatal tiene como foco de atención la unidad familiar, y lo hace en la forma de política social, con el objetivo de procurar el bienestar de la familia; desarrollándose la acción pública a través de diversas políticas desde distintas áreas de intervención (ayudas económicas, vivienda, fiscalidad, etc.). Ya sea de una forma explícita a través de departamentos, políticas, programas o medidas en la forma de "política familiar» o «política de familia», ya sea como una «dimensión familiar» subyacente a la acción del estado del bienestar, la familia, como unidad sustentada por el trabajador, es entendida como espacio al que se debe procurar bienestar e integración social en su relación con el contexto social en el que se encuentra, y respecto al mercado, en particular ${ }^{1}$.

En cambio, los nuevos riesgos suponen, no sólo proveer los servicios necesarios para el bienestar de la unidad familiar, sino también la necesidad de atender a la provisión de servicios de bienestar que tradicionalmente desarrollaban en el seno de la propia familia y, en particular, aquellos que, centrados en la atención a los dependientes, provee tradicionalmente la mujer ${ }^{2}$. Esto es,

1. En el intento de analizar este aspecto, lo común ha sido construir definiciones de politica familiar en base a su visibilidad institucional y/o la existencia de objetivos y resultados explicitos y directos. Esta perspectiva ha dado lugar a confusiones analíticas, pues, como se señala en diversos estudios, la existencia de un área específica de intervención con programas y objetivos explícitos no es condición necesaria para que se desarrollen medidas de atención a la familia, ya sea en la forma de atención a los riesgos clásicos o en la de nuevos riesgos familiares (Bahle y Maucher, 1998; Flaquer, 2002).

2. De ahí que a los riesgos sociales basados en los derechos del trabajador se denominen «riesgos clásicos» o también «riesgos masculinos», y los relacionados con deberes de cuidado y obligaciones de provisión se conozcan como «nuevos riesgos», «riesgos asociados a la familia» o también «riesgos femeninos» (Scheiwe, 1997). Sobre la distinción entre riesgos masculinos y riesgos femeninos, pueden consultarse Daly (1996) y Rolf (1989). 
la incorporación progresiva de ésta al mercado laboral enfrenta a las familias $y$, por ende, al estado, al dilema de desarrollar servicios de cuidados, de bienestar, para la población dependiente como alternativa a los desarrollados por la mujer a través del ejercicio de su rol doméstico tradicional. No se trata, pues, únicamente de la relación entre la familia y el estado, o de la primera con el mercado, sino de la forma en que se organiza la dinámica interna de la unidad familiar y el papel que el estado pueda desempeñar ofreciendo oportunidades para modificar tal dinámica (Lewis, 1992, 1997; Esping-Andersen, 1999a; Sarraceno y Naldini, 2001).

Así pues, los cambios en la familia plantean que la intervención estatal no se oriente tanto, o únicamente, a la integración sociolaboral de los trabajadores y las trabajadoras, sino, en particular, a la posibilidad de que éstas puedan conciliar la vida laboral con la vida familiar. Esto es, no se trata únicamente de integración sociolaboral, sino también de conciliación (Gornick y otros, 1997). Es desde este punto de vista que, en buena medida, se propone el concepto de desfamilización (Esping-Andersen, 1999a) como instrumento analítico para conocer la medida en que las familias tienen oportunidades para cubrir la demanda de cuidados de los dependientes sin tener que recurrir a la autoprovisión y, en particular, a la mujer.

Como es sabido, el concepto de desfamilizacion es introducido por EspingAndersen (1999a) como nuevo indicador en una revisión a su ya clásica tipología de los regímenes de bienestar ${ }^{3}$. En concreto, se refiere al grado en que los estados asumen responsabilidades de bienestar que tradicionalmente desarrollaban o desarrollan las familias, a fin de relajar o liberar a éstas de dichas obligaciones. Ello supone, por un lado, reconsiderar el papel central que desempeñan los hogares en la producción de bienestar y, por otro lado, atender a los cambios en las formas de participación femenina en el mercado de trabajo, como nuevo riesgo social sobre el que el estado puede intervenir. De esta forma, la desfamilización se referiría al grado en el que la familia, principalmente la mujer, puede dejar de absorber parte de las responsabilidades de bienestar, con lo que el esfuerzo estatal de desfamilizacion supondría una estructura de oportunidades orientada, en gran medida, a la conciliación de la vida laboral y familiar. A partir de ello, se podrían delimitar estrategias más familistas o desfamilistas por parte de los estados y los regímenes de bienestar (Esping-Andersen, 1999a; Flaquer, 2004).

3. Dicha revisión viene dada como respuesta a la crítica feminista del concepto de desmercantilizacion, el cual, y en base a dicha crítica, no considera el papel del trabajo realizado por las mujeres dentro de la familia en la conocida tipología de Esping-Andderen de los regímenes de bienestar. De esta forma, la familia pasa a ser examinada como una institución clave en el análisis del estado del bienestar, facilitando el paso de una perspectiva centrada en la relación entre estado y mercado a otra más socioestructural, en la que la familia cuenta como decition-maker, de hecho, "es un importante actor, cuyo comportamiento y decisiones influencia y es influenciado directamente por el estado del bienesar y el mercado de trabajo. Los regímenes de bienestar pueden identifiarse mucho más sistemáticamente en términos de la tríada intercausal de estado-mercado y familia» (Esping-Andersen, 2002: 35). 
Evidentemente, no sólo cabrá hablar de esfuerzo de desfamilización por parte del estado. En el marco de los sistemas de bienestar, entendidos como welfare mix, tales oportunidades pueden provenir de una nueva división de los roles de género en el seno de la familia (desfamilización en los hogares), por la existencia de oferta privada de servicios de cuidado (desfamilización a través del mercado), o bien por la existencia de políticas y medidas que las autoridades públicas desarrollen al respecto (desfamilización estatal). Esto último implicaría que la acción del estado del bienestar podría incluir acciones encaminadas a producir procesos de desfamilización, esto es, a aliviar responsabilidades de provisión de bienestar por parte de las familias. De esta forma, la desfamilización estatal consistiría en «una propuesta de colectivizar el peso y las responsabilidades de las cargas familiares, lo que representa claramente una condición necesaria para las mujeres que buscan armonizar trabajo y maternidad» (Esping-Andersen: 1999b: 277).

De hecho, hasta hace relativamente poco tiempo, el análisis del estado del bienestar, de su extensión, se centraba en una perspectiva socioeconómica y, en concreto, en el esfuerzo de gasto que realiza en términos de política social. Del mismo modo, la acción benefactora con respecto a la familia, la dimensión familiar del estado del bienestar, se solía considerar en términos de gasto en familia desde diversas áreas, políticas y departamentos, ya fuese de forma implícita o explícita, tal y como se ha indicado más arriba.

Ahora bien, esta perspectiva, aún dando cuenta del esfuerzo de desfamilización estatal, se orienta sobre todo al conocimiento del esfuerzo genérico que realiza el estado para procurar el bienestar de las familias, y podría considerarse que no da cuenta de la intensidad ni de la naturaleza de tal esfuerzo con respecto a los nuevos riesgos familiares derivados de las necesidades de conciliación. Es por ello que cabe sostener la necesidad de analizar tal esfuerzo atendiendo no sólo al conjunto de oportunidades que, mediante la política social, el estado ofrece para el bienestar de las familias, sino también las oportunidades de conciliación que de ellas se derivan o, si se prefiere, el bienestar «en las familias» (Gornick y otros, 1997).

Desde esta perspectiva, y con una finalidad analítica, la desfamilización, o el esfuerzo estatal de desfamilización, puede entenderse como el conjunto de oportunidades que la acción estatal ofrece para procurar tanto bienestar familiar (al conjunto de sus miembros), como para la conciliación de la vida doméstica y la vida familiar de sus miembros, y de la mujer en particular, dado que, tradicionalmente, es sobre ella que recaen los servicios de cuidado para con los otros miembros. Esto es, no se trata sólo de aliviar las responsabilidades de provisión de bienestar por parte de las familias en general (salud, vivienda, educación...), sino también de aliviar específicamente las del actor que principalmente los provee, para procurar con ello su integración laboral. $\mathrm{O}$, si se prefiere, analíticamente, el esfuerzo estatal de desfamilización atendería no sólo a los clásicos riesgos sociales, sino también a los nuevos riesgos familiares (Scheiwe, 1997), en la medida en que se ofrecen oportunidades públicas para que la familia externalice la provisión de servicios (Flaquer, 2002) 
sin tener que asumir, o asumir en menor medida, riesgos sociales, sean éstos tradicionales o nuevos.

\section{El esfuerzo estatal de "desfamilización»: dimensiones e indicadores básicos para el análisis comparado}

De lo expuesto en el apartado anterior, se deriva que la desfamilización, como instrumento analítico, contiene, cuando menos, tres dimensiones básicas, a saber: esfuerzo de política social, esfuerzo de servicios de atención a dependientes y esfuerzo de conciliación. Las dos últimas se pueden agrupar en términos de atención a los nuevos riesgos familiares.

La primera, referida al esfuerzo para proporcionar bienestar como integración socioeconómica, se centraría en la política social que tiene como objeto de intervención específico a la unidad familiar. En este sentido, tal y como se ha indicado más arriba, esta dimensión suele ser analizada en términos de esfuerzo económico, esto es, el gasto en política social destinado a la familia entendida como unidad de intervención, o, si se quiere, más llanamente, "gasto en familia", sin necesidad de especificar qué áreas o políticas específicas, sino más bien agrupando a las clásicas de la acción del estado del bienestar ${ }^{4}$.

Respecto a los servicios de atención a dependientes, cabe pensar en aquellos destinados a los cuidados de mayores y niños; más concretamente, la extensión y cobertura de tales servicios. La existencia de ambos servicios supone, evidentemente, una estructura de oportunidades favorable para que los hogares puedan dejar de absorber riesgos sociales en la forma de servicios de cuidado. Entre ellos, es destacable, en particular, aquellos que posibilitan la escolarización de los niños menores, pues ello suele incidir en una mejora de la incorporación de la mujer al mercado de trabajo. Se trata de una dimensión intermedia entre el esfuerzo de política social, en cuanto a política educativa, y esfuerzo de conciliación, en cuanto que ofrece una estructura pública para el cuidado de los menores (Levy y otros, 2002), pues esta dimensión se orienta tanto a ofrecer oportunidades para aliviar responsabilidades de bienestar familiar en términos de integración socioeconómica (por el lado de la integración escolar para unos miembros de la familia $)^{5}$, como a hacerlo en térmi-

4. Así, por ejemplo, en referencia a esta dimensión, Esping-Andersen (1999a) utiliza el indicador de gasto público en servicios a la familia (\% PIB), para la medición de la desfamilización en diferentes regímenes de bienestar (Esping-Andersen: 1999: 61). Igualmente, en otros trabajos, como el proyecto Family Change and Family Policies in the West, dirigido por P. Flora, S. Kamerman y A. Kahn, se recoge de forma sistemática esta dimensión a través de distintas medidas de gasto estatal en ayuda o atención a la familia, bajo diferentes conceptos incluidos en las política sociales nacionales. Algunos resultados de este proyecto pueden consultarse en Pfenning y Bahle (2000).

5. Esta dimensión es utilizada, entre otros, por Esping-Andersen(1999a) a través de un indicador de cobertura de centros de atención para niños de 0 a 3 años; del mismo modo hacen Gornick y otros (1997) y Flaquer (2004), desagregando el grupo de edad susceptible de beneficiarse de este esfuerzo en dos grupos: de 0 a 3 años, por un lado, y de 3 a 6 años, por otro. 
nos de posibilidades de integración laboral, como conciliación (para otros miembros de la familia).

Ahora bien, específicamente, el esfuerzo de conciliación podría conocerse, de forma directa, a través de medidas concretas destinadas a favorecer la incorporación y/o reincorporación de mujeres con cargas familiares, ya sea en la forma de permisos de maternidad (y/o paternidad), pagados o no, ya sea en la forma de permisos o ayudas para el cuidado de niños, con independencia de la existencia de centros que permitan su atención y escolarización. De hecho, algunos estudios han mostrado que la existencia de tales medidas ejerce un efecto positivo sobre el empleo de las madres trabajadoras (Forséen y Hakovirta, 2000; Gornick y otros, 1997), y actúan como incentivos, pues reducen el conflicto de las mujeres con su rol familiar tradicional, al permitirles, especialmente a las madres con hijos, permanecer activas (Mandel y Semyonov, 2003). Esto es, pueden ser consideradas como indicador del esfuerzo de conciliación estatal y, con ello, como uno de los componentes del esfuerzo público de desfamilización.

En su conjunto, cabría, pues, delimitar dos grandes dimensiones respecto al esfuerzo público de desfamiliazción. Por un lado, la dimensión de política social orientada a intervenir sobre riesgos sociales clásicos, esto es, a aliviar la provisión de servicios de bienestar en el seno de la familia relacionada con los modos más clásicos de intervención del estado del bienestar o, si se prefiere, a garantizar la integración de la familia, en su conjunto, al marco socioeconómico en el que se sitúa. Por otro lado, la dimensión de «atención a nuevos riesgos familiares», orientada a intervenir sobre tales riesgos y, con ello, en la configuración interna de la familia como unidad provisora de servicios, en la que cabría diferenciar una subdimensión orientada en específico a proporcionar oportunidades de cuidado para los dependientes, los niños en particular, o dimensión de cuidado de dependientes, por un lado, y las orientadas a procurar la conciliación entre vida laboral y doméstica para los hogares, y en particular, las mujeres con cargas familiares, o dimensión de conciliación, por otro (tabla 1).

Delimitado de esta forma, el concepto de desfamilización, no se refiere tanto a las áreas de intervención, en la forma de política familiar, sino a una

Tabla 1. Esfuerzo público de desfamilización: dimensiones básicas

\begin{tabular}{lll}
\hline Dimensión & Subdimensión & Medidas \\
\hline Política social & Económica & Gasto público en familia \\
\hline Atención a nuevos riesgos & Cuidado de dependientes & $\begin{array}{l}\text { Servicios públicos de atención } \\
\text { a niños y mayores }\end{array}$ \\
\cline { 2 - 3 } & Conciliación & Permisos de maternidad \\
\cline { 3 - 3 } & & Permisos para cuidados \\
\hline
\end{tabular}

Fuente: elaboración propia. 
dimensión analítica que da cuenta de la medida en que el estado se esfuerza por ofrecer oportunidades para que las familias puedan dejar de proveer servicios a sus miembros o, si se prefiere, relajen la absorción de riesgos sociales, tradicionales o nuevos ${ }^{6}$. Cabe pensar que este concepto analítico sea susceptible de medición a través de indicadores concretos, tal y como se propone en los siguientes apartados para el caso de quince países de la Unión Europea durante el periodo 1970-1990.

\subsection{Indicadores para la medición del esfuerzo público de desfamilización}

Las dimensiones y subdimensiones señaladas pueden orientar el proceso de elaboración de un indicador de esfuerzo estatal o público de desfamilización que permitan el desarrollo de análisis comparados - tanto longitudinal, uno o varios países en el tiempo, como comparaciones entre países-. Además de la cuestión obvia referida a la existencia de datos, los indicadores que se deben utilizar para realizar tal ejercicio tendrían que cumplir, cuando menos, las siguientes condiciones:

1) Debe tratarse de un tipo específico de ayuda o servicio público orientado a incentivar la desfamilización en alguna de sus dimensiones. Debe medir, pues, un aspecto de la acción que esté bajo control público, es decir, asumida como esfuerzo o medida pública (estatal) y que suponga una menor absorción de riesgos sociales, de cualquier tipo, por parte de las familias.

2) En el caso de la dimensión de atención a nuevos riesgos familiares, el indicador debe capturar rasgos de la acción pública que se prevé puedan afectar a las decisiones que sobre el empleo realicen las mujeres. Esto es, los indicadores deben reflejar incentivos que afecten a las decisiones de incorporación o reincorporación al trabajo por parte de las mujeres con cargas familiares.

3) Los indicadores deben ser exógenos a las decisiones de empleo individuales, es decir, debe tratarse de medidas que sean independientes o antecedentes a las decisiones de empleo, pues deben medir la estructura de oportunidades que ofrece la acción pública, con independencia de que éstas sean o no consideradas o empleadas por la ciudadanía.

Atendiendo a estos criterios, los indicadores seleccionados se han tomado de dos bases de datos que ofrecen información sobre medidas públicas orientadas a la oferta de oportunidades de desfamilización en veintidós países entre

6. Como concepto analítico, la desfamilización representaría una aportación tanto al debate sobre los rasgos y las características de las políticas familiares en cada país, como sobre la dimensión familiar del estado del bienestar, en el sentido de que permite captar empíricamente el esfuerzo público de atención a las familias, a través de la medición del grado y de la intensidad del esfuerzo público en diferentes contextos nacionales, así como su evolución en el tiempo. 
1970 y $1990^{7}$. En concreto, se han seleccionado tres conjuntos de indicadores para quince países de la Unión Europea, a saber: gasto público en familia, extensión de la escolarización de niños menores de seis años y diversas medidas de conciliación. Dichos indicadores se muestran en la tabla 2.

En concreto, el gasto se refiere al porcentaje que supone el gasto público en familia sobre el producto interior bruto de cada país y para cada año considerado $^{8}$. La extensión de la escolarización se refiere al porcentaje de niños y niñas menores de seis años que están escolarizados, esto es, hasta el momento previo a la escolarización obligatoria. Este indicador consistiría en un esfuerzo adicional al de la política educativa obligatoria, con lo cual se puede medir el esfuerzo estatal que se orienta a ofrecer cuidados.

Evidentemente, debe considerarse como una aproximación a la dimensión de «cuidados a los dependientes», porque no se considera la oferta como tal, sino el hecho de que sea utilizado por la población de referencia y, por otro, no se incluye la atención al otro importante grupo de dependientes, los mayores. Ahora bien, a diferencia de otras fuentes ${ }^{9}$, este indicador existe para todos

Tabla 2. Esfuerzo público de desfamilización: dimensiones, indicadores y descriptivos para UE15

\begin{tabular}{lllll}
\hline Dimensión & Indicador & Medidas & Media para UE15 & Desv. típica \\
\hline Política social & Gasto público & $\begin{array}{l}\text { Ayuda familiar } \\
\text { (\% PIB) }\end{array}$ & 25,7 & 17,8 \\
\hline $\begin{array}{llll}\text { Atención a } \\
\begin{array}{l}\text { nuevos riesgos } \\
\text { familiares }\end{array}\end{array}$ & Conciliación & $\begin{array}{l}\text { Permisos } \\
\text { de maternidad } \\
\text { Permisos para }\end{array}$ & 46,5 & 16,07 \\
& Atención Escolar & $\begin{array}{l}\text { Niños escolarizados } \\
\text { en preprimaria } \\
\text { (\% del grupo de edad) }\end{array}$ & 58,2 & 23,8 \\
\hline
\end{tabular}

Fuente: elaboración propia a partir de datos de Gauthier y Bortnik (2001) y Gauthier (2003). Los indicadores se han estandarizado en escala [0 a 100], siendo cero el valor mínimo de los quince países y cien, el valor máximo.

7. Las bases de datos utilizadas han sido elaboradas bajo la dirección de A. H. Gauthier, responsable del servicio de investigación en Política Pública Comparada, Departamento de Sociología de la Universidad de Calgary (Canadá). Se trata, concretamente, de GAUTHIER A. H.; BortniK, A. (2001). Comparative Maternity, Parental, and Childcare Database, Versión 2 y Gauthier, A. H (2003). Comparative Family Benefits Database, Versión 2. Agradecemos a A. H. Gauthier las aclaraciones realizadas en relación con las medidas contenidas en ambas bases de datos y la metodología empleada en su construcción.

8. Se trata del concepto «ayuda familiar», cuyo contenido difiere en cada país (prestaciones por hijo, subvenciones por vivienda, reducciones fiscales, ayudas para mantenimiento de ingresos del hogar, etc.). Para más detalle, puede consultarse Gauthier (2001 y 2003).

9. Como, por ejemplo, las diferentes bases de datos elaboradas por la OCDE para diversos años. 
los países y en el periodo señalado, y aunque menos sensible a diferencias en el tiempo y entre países, mide el esfuerzo de atención a dependientes, pues se trata de niños en edad de escolarización no obligatoria ${ }^{10}$.

Respecto a las medidas de conciliación, se han considerado tanto los permisos de maternidad, como los permisos para cuidados. Los primeros se refieren a las licencias por maternidad o adopción obigatorias como derecho de las trabajadoras, que se traducen en duración y prestación económica; y los segundos se refieren a licencias y excedencias laborales concedidas a los padres y a las madres posteriores al nacimiento de los hijos, con la finalidad explícita de prestar atención y cuidados a los menores; dichas licencias suponen un abandono temporal del puesto de trabajo, con o sin prestación económica. Puesto que lo que se pretende medir es la intensidad del esfuerzo público en esta dimensión, ésta podría calibrarse en referencia a su duración (en semanas), a la compensación económica (en tanto por ciento del salario femenino) o a ambas a la vez, tal y como han sido aquí consideradas para la construcción del indicador en cada caso, con la finalidad de homogeneizar la medida conjunta del esfuerzo en conciliación en términos de intensidad.

Mediante la agregación de estos indicadores, podría elaborarse un indicador de esfuerzo público de desfamilización (IEPD) y, sobre todo, indicadores que midan sus dimensiones básicas: indicador de esfuerzo de gasto (IEG), indicador de esfuerzo de conciliación (IEC) e indicador de esfuerzo de atención a niños o de escolarización (IEE), éste último como aproximación a la dimensión de atención a los dependientes.

Ahora bien, para permitir el desarrollo de ejercicios comparativos, se ha procedido a su estandarización siguiendo la propuesta técnica de Gornick y otros (1997), por la que se hace igual a 0 el valor más bajo de cada indicador y a 100 el valor más alto del periodo y del país considerados. Mediante este ejercicio de estandarización, no sólo se mediría la intensidad del esfuerzo público, sino que también se contextualizaría en el marco de los países y del periodo temporal objeto de análisis, además de permitir una fácil interpretación. Vendría a constituir, pues, una escala equivalente acerca de la oferta de oportunidades de desfamilización estatal, con un recorrido estándar igual al intervalo $[0,100]$.

10. En distintos estudios (Gornick y otros, 1997; Flaquer, 2004), se señala la necesidad de diferenciar, dentro del grupo de población en edad preescolar (0-6 años), el grupo de menos de tres años y el grupo de más de tres. Aquí se presenta como grupo único después de haber comprobado la validez del indicador - porcentaje de niños de 0 a 6 años escolarizados - para todos los países y en cada año. Concretamente, utilizando otras fuentes (OCDE, 2001) que diferencian los dos segmentos y poniéndolos en relación con el grupo de 0 a 6 años, en todos los casos la correlación es positiva, ya sea teniendo en cuenta el grupo de $<3$ años $(r=0.13)$, el grupo de $>3$ años $(r=0.59)$ o ambos sumados $(r=0.50)$. 


\section{El esfuerzo público de desfamilización en la Unión Europea (1970-1999): dimensionalidad, cambio en el tiempo y diferencias entre grupos de países}

Para conocer la validez de los indicadores propuestos para el conjunto de los quince países de la Unión Europea durante el periodo 1970-1999, se realizará un análisis factorial que permita confirmar la existencia de las dimensiones delimitadas, atendiendo, asimismo, a su consistencia en el tiempo y entre países, como también un breve análisis descriptivo para constatar que los indicadores reflejan procesos de cambio y diferencias entre países. Esto es, diversas pruebas para dar cuenta de la validez de la propuesta de medición realizada.

\subsection{Las dimensiones del esfuerzo público de desfamilización: la importancia creciente de la conciliación}

A tenor de la propuesta planteada acerca de las dimensiones del esfuerzo público de desfamilización, la aplicación de análisis factorial de componentes principales al conjunto de países para todo el periodo de tiempo debería producir tres factores. No obstante, tal y como puede apreciarse en la tabla 3, los resultados muestran la existencia de dos factores que explican algo más del $70 \%$ de la varianza existente entre los países para el periodo considerado.

En concreto, los factores parecen diferenciar, sobre todo, entre las dos dimensiones de atención a riesgos familiares, esto es, conciliación y escolarización, respectivamente, pues el gasto público aparece ligado a ambas. Esto vendría a mostrar, por un lado, la existencia de diferencias entre la estrategia de conciliación y la de atención escolar, así como también la relación existente entre el esfuerzo orientado a atender riesgos sociales clásicos con el que se orienta hacía los nuevos riesgos familiares.

Ello aconsejaría la distinción de las tres dimensiones señaladas, pues el gasto no aparece específicamente asociado a ninguna de las otras en particular, esto

Tabla 3. La dimensionalidad del esfuerzo público de desfamilización: países de la Unión Europea entre 1970-1999

\begin{tabular}{llrr}
\hline & & \multicolumn{2}{l}{ Factores } \\
\cline { 3 - 4 } Dimensiones & Indicadores & $\mathbf{1}$ & \multicolumn{2}{l}{$\mathbf{2}$} \\
\hline Gasto público & Gasto público en ayuda familiar & 0,638 & 0,538 \\
\hline De conciliación & Permisos de maternidad & $\mathbf{0 , 8 3 4}$ & 0,211 \\
& Permisos para cuidados & $\mathbf{0 , 7 8 6}$ & $-0,166$ \\
\hline De escolarización & Niños en educación preprimaria & 0,047 & $\mathbf{0 , 9 1 0}$ \\
\hline Varianza explicada & 44,7 & 26,4 & \\
\hline Varianza acumulada & 44,7 & $\mathbf{7 1 , 2}$ & \\
\hline
\end{tabular}

Fuente: elaboración propia a partir de resultados de análisis factorial. Método de extracción: análisis de componentes principales. Metodo de rotación: normalización Oblimin con Kaiser. 
es, mide el esfuerzo de política social que realiza el estado respecto a la familia, de lo que se deriva que el esfuerzo de atención a nuevos riesgos familiares, aunque muestre diferencias entre una estrategia centrada en la conciliación y otra en la atención a menores, se encuentra estrechamente relacionado con el esfuerzo económico que se realiza para atender a las familias. $\mathrm{O}$, si se quiere, que la atención a nuevos y tradicionales riesgos familiares no se muestran empíricamente como esfuerzos públicos independientes.

Como puede apreciarse en el gráfico 1 , los tres indicadores elaborados muestran una tendencia creciente para el conjunto de los países de la Unión Europea. No obstante, el indicador de conciliación muestra una pendiente de crecimiento más alta que los otros dos y, sobre todo, a partir de mediados de los años ochenta. De esta forma, básicamente, los resultados concuerdan con la literatura existente al respecto, en el sentido de que la atención a los nuevos riesgos a través de distintas medidas de conciliación representa una nueva estrategia de intervención sobre la familia y parte central del esfuerzo público de atención a la misma, que debería tenerse en cuenta en el análisis del desarrollo reciente del estado del bienestar ${ }^{11}$.

\section{Gráfico 1}

Cambios en el esfuerzo público de 'des-familización' en la Unión Europea (1970-1999)

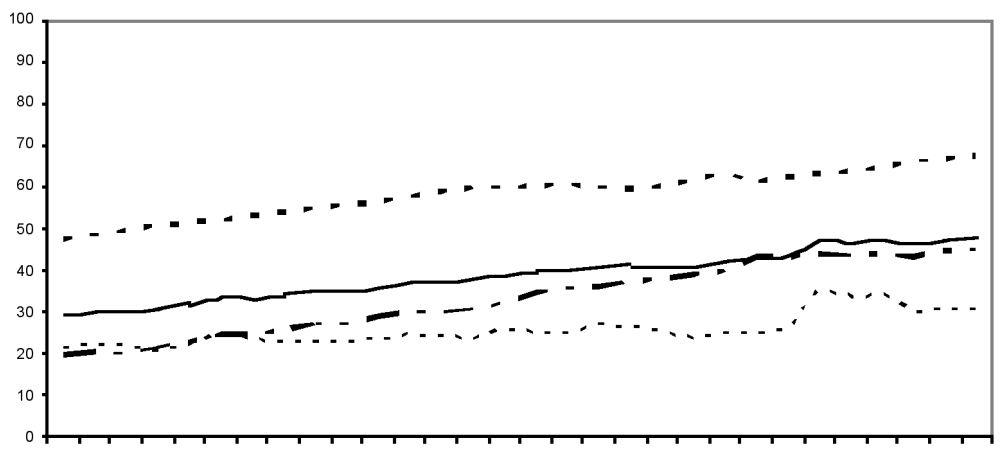

— Indicador de Esfuerzo Público de Des-fam ilización

- - - Indicador de Gast o en Fam ilia

- Indicador de Conciliación

- - Indicador de Atención Escolar

Gráfico 1. Cambios en el esfuezo público de desfamilización en la Unión Europea (1970-1999). Fuente: elaboración propia a partir de datos de Gauthier y Bortnik (2001) y Gauthier (2003).

11. De hecho, un nuevo patrón de relación con el empleo de las mujeres viene representado por la continuidad en el mismo después del nacimiento de los hijos (Gornick y otros, 1997), de lo que se deduce que la existencia de medidas de conciliación puedan estar constituyendo un importante incentivo en tal sentido (Forssén y Hakovirta, 2000; Mandel y Semyonov, 2003). 
Por lo demás, estas tendencias apuntan a lo mostrado por el análisis factorial, esto es, la relación existente entre el gasto y las otras dos dimensiones (una asociación positiva). Pero también revela la diferenciación entre las dos dimensiones referidas a los nuevos riesgos familiares, esto es, que el crecimiento en la extensión de las medidas de conciliación avanza más rápido que las de escolarización a partir de mediados de los años ochenta. De hecho, tal y como se muestra en el gráfico 2, la correlación entre los indicadores disminuye al controlarse por el tiempo y, sobre todo, lo hace entre conciliación y escolarización. Esto viene a revelar la importancia de atender a la dimensión de conciliación como dimensión del esfuerzo público de desfamilización. Pero, además, también viene a confirmar la validez de los indicadores, pues la dimensionalidad entre ellos permanece estable en el tiempo; esto es, se trata de mediciones que, dando cuenta de diferentes dimensiones, muestran igualmente que éstas y la estructura básica de relación entre ellas permanecen estables en el tiempo ${ }^{12}$.

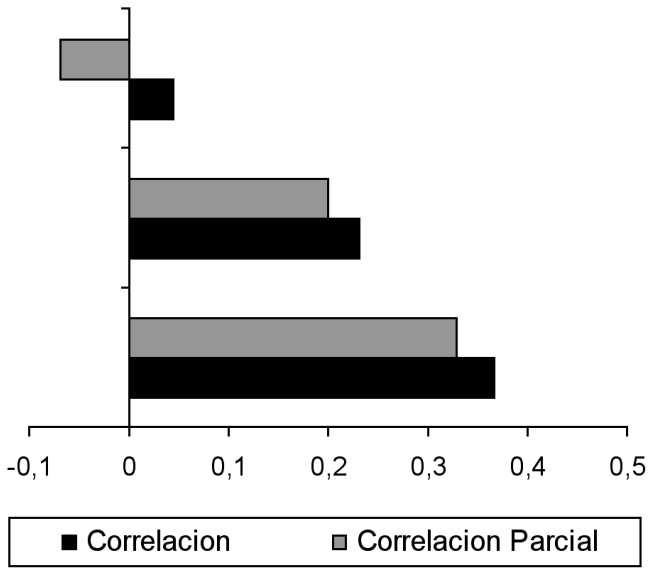

Gráfico 2. Relaciones entre dimensiones de desfamilización. Correlaciones simples y parciales controladas por el año. Fuente: elaboración propia a partir de datos de Gauthier y Bortnik (2001) y Gauthier (2003).

12. Como prueba de frabilidad, se ha realizado un análisis factorial de todas las dimensiones considerando tres periodos de tiempo (1970-1979: 1980-1989; 1990-1999). Las saturaciones de los indicadores (puntuaciones factoriales) son similares en los tres periodos. 


\subsection{Las diferencias entre paises: sobre estructuras de bienestar consolidadas y atención a nuevos riesgos}

Ahora bien, además del cambio en el tiempo, los indicadores permiten mostrar diferencias entre países. En el gráfico 1 se ha mostrado la evolución del esfuerzo público de atención a la familia (IEPD) en quince países de la UE. En este apartado, se atenderá a las tendencias de crecimiento más o menos intensas de cada dimensión del IEPD según los países ${ }^{13}$.

Como puede observarse en el gráfico 3, cabría hablar de un patrón constante en la intensidad del esfuerzo público de atención a la familia, según el cual los países del centro y del norte de Europa se diferencian claramente de los países del sur, más parecidos a su vez al Reino Unido e Irlanda. No obstante, la tendencia común de los quince países de la Unión Europea es de crecimiento lineal en el esfuerzo de desfamilización, con un momento crítico o de inflexión en el tiempo que se corresponde con la década de 1980, si bien el IEPD muestra que es en los países del centro de Europa donde esta tendencia es más estable a la vez que intensa.

Gráfico 3

El esfuerzo publico de 'des-familización' (1970-1999)

Evolución e intensidad por grupos de países

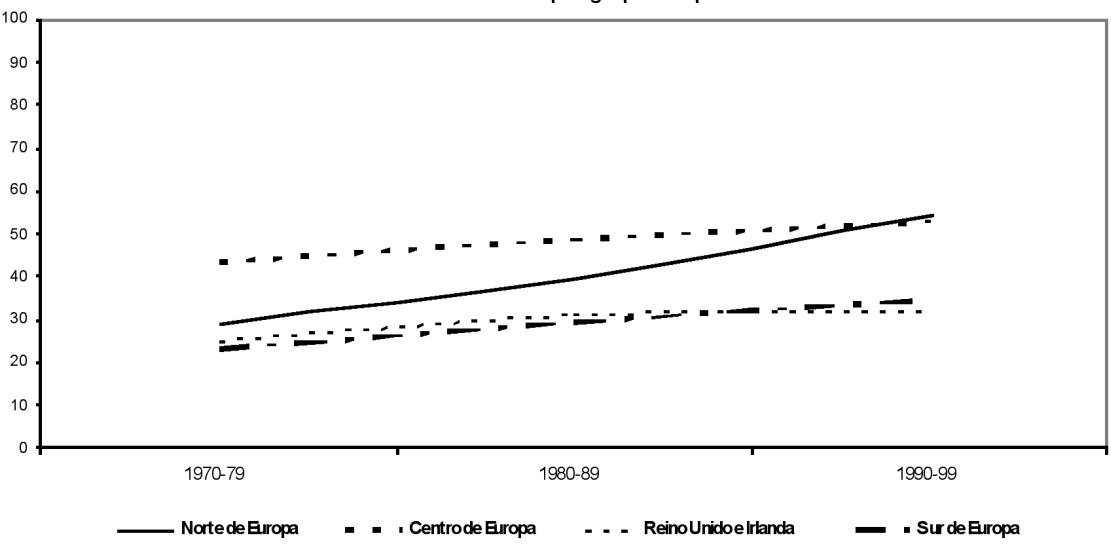

Gráfico 3. El esfuerzo público de desfamilización (1970-1999). Evolución e intensidad por grupos de países. Fuente: elaboración propia a partir de datos de Gauthier y Bortnik (2001) y Gauthier (2003).

13. Por razones de espacio y para mayor claridad en la exposición, se han agrupado los países, correspondiendo cada grupo a una zona geográfica de la UE: norte de Europa (Dinamarca, Suecia y Finlandia), centro de Europa (Bélgica, Holanda, Luxemburgo, Francia, Alemania y Austria), sur de Europa (España, Italia, Portugal y Grecia), y un cuarto grupo compuesto por el Reino Unido e Irlanda. 
Ello concuerda, como es sabido, con la existencia de una continuada tradición de atención a la familia en este grupo de países a lo largo del periodo analizado, lo cual permite afirmar que se trata de un componente consolidado en sus estructuras de bienestar y, principalmente, de política social (Gauthier, 1996; Hantrais y Letabrier, 1995, 1996).

Así, en relación con las dimensiones que componen el IEPD (gráficos 4, 5 y 6), mantienen la tendencia en la estabilidad del esfuerzo principalmente en el gasto y en atención escolar, lo que apunta a que el esfuerzo público de atención a la familia en los países del centro de Europa consiste en una estrategia de intervención centrada principalmente en el modelo de familia de tipo male breadwinner, es decir, la familia como unidad receptora y bajo una lógica salarial, esto es, las prestaciones son destinadas a la unidad familiar a través del varón cabeza de familia (Lewis, 1992, 1997; Hantrais y Letabirer, 1995, 1996).

En cualquier caso, los indicadores muestran la importancia concedida a la atención y al bienestar de la familia en los países del centro de Europa y se puede observar que ello se concreta en estrategias estatales estables y consolidadas $^{14}$.

Grafico 4

El gasto en familia: estructuras del bienestar y riesgos clásicos

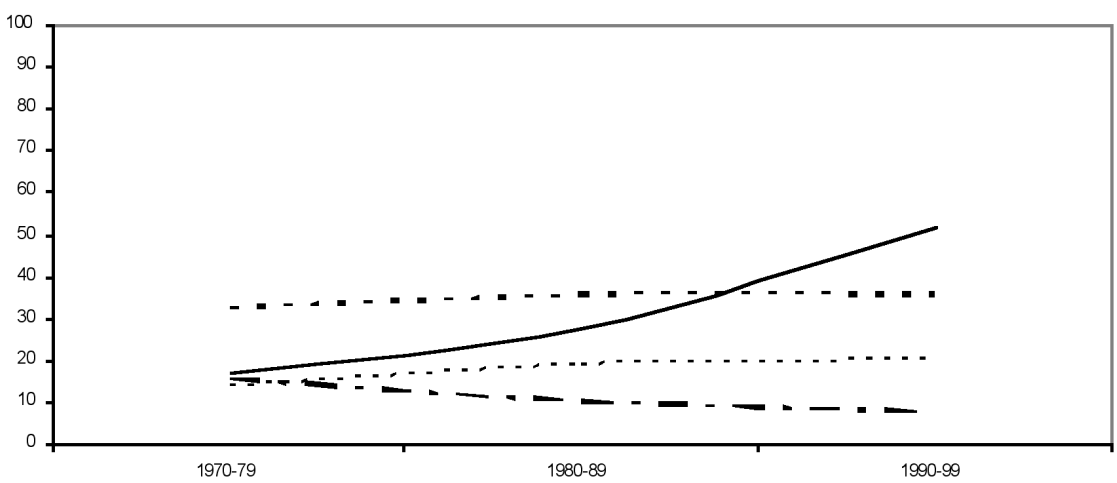

Norte deEuropa - - Centrode Europa - - . ReinoUnidoe rtanda - -Surde Europa

Gráfico 4. El gasto en familia: estructuras del bienestar y riesgos clásicos. Fuente: elaboración propia a partir de datos de Gauthier y Bortnik (2001) y Gauthier (2003).

14. Cabe añadir que en países como Francia, Bélgica, Luxemburgo, Alemania o Austria, el desarrollo de medidas de atención a la familia a través de la política social acompaña al propio desarrollo del estado del bienestar. A este respecto, puede consultarse a Gauthier (1996), Hantrais y Letabrier (1995, 1996), Sarraceno y Naldini (2001), y Morgan (2002), entre otros. 


\section{Grafico 5}

Estrategia de Conciliación en la UE (1970-1999)

Estado, individuo y familia

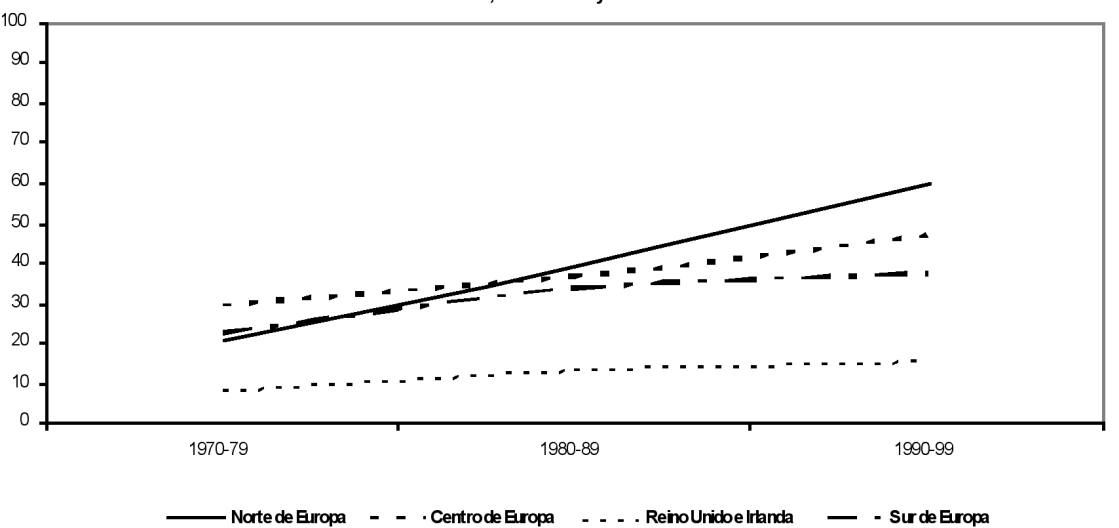

Gráfico 5. Estrategia de conciliación en la UE (1970-1999). Estado, individuo y familia. Fuente: elaboración propia a partir de datos de Gauthier y Bortnik (2001) y Gauthier (2003).

Gráfico 6

La Atencion Escolar: atención consolidada a la familia

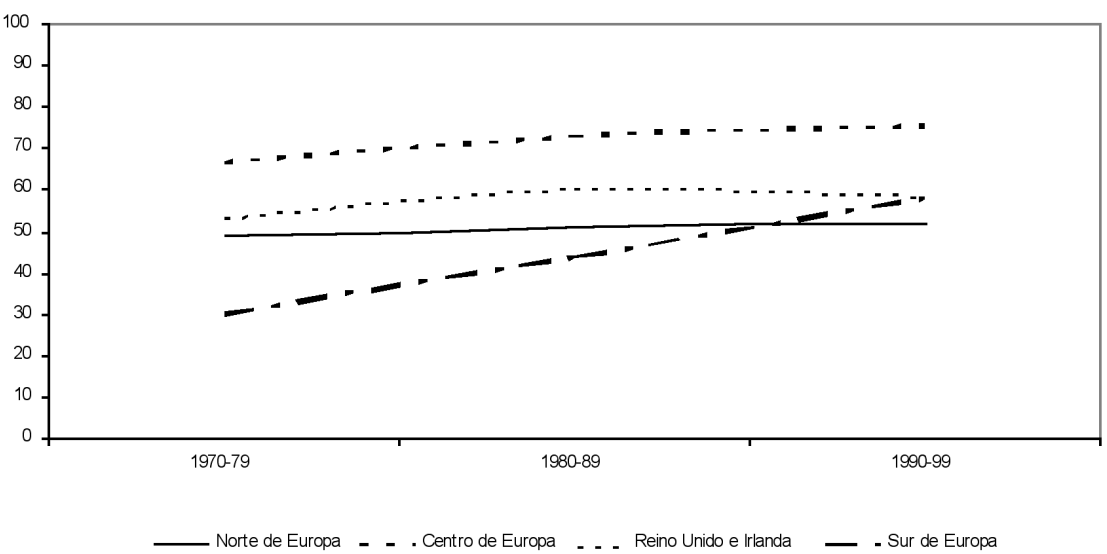

Gráfico 6. La atención escolar: atención consolidada a la familia. Fuente: elaboración propia a partir de datos de Gauthier y Bortnik (2001) y Gauthier (2003).

En cambio, los países del norte de Europa (Dinamarca, Suecia y Finlandia) muestran una dinámica menos estable $\mathrm{o}$, si se prefiere, el esfuerzo público de atención a la familia podría definirse, entre otros aspectos, en relación con los cambios experimentados en su intensidad entre 1970 y 1999. Como se obser- 
va en la evolución del indicador de esfuerzo público de desfamilización, del indicador de esfuerzo en gasto y, sobre todo, del indicador de esfuerzo en conciliación, el rasgo más destacado es el aumento en la tendencia de crecimiento a partir de la década de 1980, lo cual se refleja tanto en el IEPD (gráfico 3), como en el esfuerzo en gasto (gráfico 4). Ambos se consolidan a lo largo de los años ochenta y noventa.

Ahora bien, en cuanto al esfuerzo en conciliación, este aumento se produce con anterioridad a los años ochenta y sigue una línea constante cuya tendencia muestra que los países del norte de Europa, junto con el gasto, han consolidado igualmente la dimensión o la estrategia de conciliación como principal componente del esfuerzo estatal desfamilizador, esto es, la atención a los nuevos riesgos familiares junto con la atención a los riesgos clásicos. $\mathrm{O}$, dicho de otra forma, las dimensiones en las que mejor se refleja la consistencia del esfuerzo público de desfamilización en los países del norte de Europa son el gasto y la conciliación, siendo la segunda en la que destaca frente a los otros grupos de países.

Teniendo en cuenta que el esfuerzo en conciliación se basa en las necesidades de las madres trabajadoras y que a los países del norte de Europa se les atribuye el desarrollo de amplias estructuras de bienestar sobre un principio de redistribución universal, la dinámica que muestra el indicador de esfuerzo en conciliación viene a demostrar que se trata de una estrategia central en el esfuerzo público de desfamilización que se basa en la lógica de los derechos y las autonomías individuales, esto es, favorecedora de mayor independencia del individuo con respecto a la familia o, lo que significa lo mismo, del apoyo estatal a las familias de dos trabajadores basado en la absorción por parte del estado de responsabilidades de bienestar; ello los diferencia del grupo de países del centro de Europa (Esping-Andersen, 1999; Hilamo, 2002),

A tenor de lo reflejado en el gráfico 3, los países del sur de Europa, así como el Reino Unido e Irlanda, no se diferencian tanto por las estrategias que desarrollan, sino por la intensidad del esfuerzo que realizan, de lo que podría deducirse que, en términos generales, serían menos desfamilizadores. No obstante, la tendencia del IEPD es igualmente la de un crecimiento lineal, siendo éste más acusado en los países del sur de Europa, donde el mayor crecimiento se produce en la estrategia de atención escolar. A este respecto, debe señalarse que uno de los factores a tener en cuenta en el proceso de desarrollo de los sistemas de bienestar en estos países, consiste en la extensión y la consolidación de la educación universal como derecho de la ciudadanía, de lo que se deriva, en relación con la importancia de este indicador en los países del sur de Europa, que se trata de una estrategia genérica de bienestar que contribuye al proceso de desfamilización de forma indirecta ${ }^{15}$, lo cual permite a los países del sur de Europa incorporar de forma constante — aunque indirecta- un aspecto de

15. En este sentido, para el caso español, puede consultarse, por ejemplo, el trabajo de Flaquer (2004). 
la dimensión de nuevos riesgos familiares como estrategia (no explícita) de desfamilización.

Por otro lado, los países del sur de Europa experimentan un crecimiento destacado en conciliación en la década de 1980 (que se corresponde con el momento de incorporación de Grecia, España y Portugal a la Unión Europea), y se estabiliza en la década de 1990, por lo que cabe pensar que el desarrollo de la estrategia de conciliación se corresponde con la necesidad de desarrollar medidas de adaptación a principios rectores supranacionales en materia de igualdad laboral ${ }^{16}$. A su vez, el menor esfuerzo de gasto en familia, e incluso su descenso, apunta a que se trata de estrategias estatales menos desfamilizadoras o, si se prefiere, en los países del sur de Europa, la familia absorbe gran parte de los riesgos sociales (Flaquer, 2002, 2004; Naldini, 2003).

En el caso del Reino Unido e Irlanda, aunque también presentan una tendencia creciente, más intensa en la década de los ochenta, se evidencia, sobre todo, un nivel bajo en esfuerzo de conciliación. Ello contrasta con el comportamiento de ambos países en la dimensión de gasto en familia, que siendo, en general, más bajo que en los países del norte y del centro de Europa (gráfico 4), muestra una tendencia estable. De ello se puede derivar que desarrollan mayor esfuerzo desfamilizador en la atención a los riesgos clásicos que en la de nuevos riesgos familiares. Más concretamente, como es sabido, en el caso del Reino Unido, la atención a la familia a través de la política social se basa en la intervención estatal ante situaciones de pobreza, exclusión, etc., es decir, riesgos clásicos, de manera que los nuevos riesgos sociales son absorbidos por la familia, con el consiguiente grado de dependencia y coste de oportunidad que ello representa, principalmente para las mujeres (Lewis, 1992, 1997).

En función de los resultados mostrados, cabe decir que el IEPD y sus componentes permiten captar, de forma aproximada y sintética, algunos aspectos del esfuerzo público de atención a la familia en la UE desde 1970 hasta 1999 y que, en términos generales, los indicadores construidos se muestran válidos y útiles, pues permiten identificar la intensidad de cada dimensión del esfuerzo público de atención a la familia según los países, así como analizar dinámicas más concretas.

\section{A modo de conclusiones}

Como se ha visto, los resultados alcanzados a través del análisis descriptivo, han permitido presentar algunos aspectos generales y otros más específicos del esfuerzo público de desfamilización en quince países europeos. En términos generales, los análisis muestran que el crecimiento del esfuerzo desfamilizador es constante en todo el periodo analizado, aunque con diferencias en cuanto a

16. Concretamente, distintas directivas europeas que, centradas en la necesidad de que exista una convergencia europea en materia de igualdad laboral, han incentivado en los países del sur de Europa la ampliación de la duración de los permisos de maternidad, así como la extensión de los permisos parentales para cuidados. 
sus dimensiones, así como la intensidad de éstas según grupos de países. Con ello, las pruebas empíricas presentadas muestran, por una parte, evidencias sobre la validez y la utilidad de los indicadores elaborados, en el sentido en que han permitido mostrar que el esfuerzo público de desfamilización es un objeto susceptible de medición, así como que los indicadores elaborados permiten realizar comparaciones en el tiempo y en el espacio que parecen corresponderse con tendencias en el tiempo y diferencias entre países que son señalados en la literatura de referencia. Por otra, pudieran estar evidenciando la potencialidad analítica del IEPD y sus componentes, pues, como se ha visto, cabría diferenciar dos aspectos básicos en el esfuerzo de desfamilización: su intensidad y el tipo o los tipos de estrategias desarrolladas.

Básicamente, la intensidad daría cuenta del grado que alcanza el esfuerzo desfamilizador en los diferentes países; como se ha visto, menor en los países del sur de Europa y en el Reino Unido e Irlanda, y mayor en los países del centro y del norte de Europa. Ahora bien, el análisis comparado podría estar revelando, asimismo, la existencia de diferentes estrategias u orientaciones en tal esfuerzo. Así, parecería que el esfuerzo en los países del norte de Europa se concentra, progresivamente, en los nuevos riesgos familiares a través de la estrategia de conciliación, en torno a una lógica de intervención universalista que toma como foco al individuo en el seno de la familia. En este aspecto, se diferenciaría del grupo de países centroeuropeos, que, aún desarrollándola, parecen destacar en mayor medida por una lógica de intervención que, asentada previamente, se centra en mayor medida en la unidad familiar, a pesar de la similitud de ambos grupos de países en cuanto a la intensidad del esfuerzo en su conjunto. De la misma forma, cabría diferenciar a los países del sur de Europa del Reino Unido e Irlanda, pues éstos últimos parecen mostrarse más atentos a los riesgos clásicos contenidos en la política social y menos a los nuevos riesgos a través de la conciliación, aunque ambos muestran un esfuerzo global menor en comparación con los países del centro y del norte de Europa.

Lo anterior revelaría que, además del esfuerzo en general, el desarrollado en cuanto a conciliación aparece como un elemento distintivo, ya sea porque es el que muestra una pauta de crecimiento más intensa, sobre todo a mediados de los años ochenta, ya sea porque, al compararlo con el más clásico de política social (como el gasto o la escolarización), podría permitir conocer la orientación o la estrategia del esfuerzo de desfamilización, de lo que cabría derivar la conveniencia de incluirlo en los análisis comparados sobre las oportunidades de desfamilización que ofrecen los estados.

Por último, es importante señalar que los resultados mostrados deben entenderse como un ejercicio por el que evidenciar el grado de validez y utilidad de la operacionalización del concepto de desfamilización y sus dimensiones. No se ha tratado de clasificar a los países o de elaborar tipologias, sino de comprobar que los indicadores pueden medir de forma realista la extensión y la intensidad del esfuerzo público de atención a la familia, el cambio en el tiempo y las diferencias entre países. Evidentemente, lo presentado no pretende más que hacer una propuesta tentativa para avanzar en el análisis de los procesos 
de desfamilización y, en particular, respecto a su medición, sus componentes básicos e indicadores susceptibles de uso para su medición. Por supuesto, cabe realizar mejoras en ello u otros ejercicios analíticos y empíricos orientados a su validación y, con ello, su aplicación a análisis comparados.

\section{Referencias bibliográficas}

Bahle, T.; Maucher, M. (1998). Developing a family policy database for Europe. Mannheim: Mannheim Centre for European Social Research.

BRADSHAW, J. y otros (1993). «A comparative study of child support in fifteen countries». Journal of European Social Policy, 3 (4): 255-271.

Bonnar, D. (1990). «The Place of Caregiving Work in Contemporary Societies». En: Hyde, J.; Essex, M. Parental Leave and Child Care. Setting a Research and Policy Agenda. Filadelfia: Temple University Press, p. 192-207.

Braun, M. (1998). «Gender Roles». En: VAn Deth, J. Comparative Politics. The problem of equivalence. Londres: Routledge, p. 111-134.

BraVo, E. (1990). «Family Leave: The Need for a New Minimun Standard». En: Hyde, J.; Essex, M. Parental Leave and Child Care. Setting a Research and Policy Agenda. Filadelfia: Temple University Press, p. 165-176.

Burns, N. S. K.; Verba, S. (2001). The Private Roots of Public Action. Gender Equality and Political Participation. Londres: Harvard University Press.

Castles, F.; Ferrera, M. (1993). «Why Divorce Rates Differ: Law, Religious Belief and Modernity». En: CASTLES F. (eds.). Family of Nations: Patterns of Public Policy in Western Democracies. Aldershot: Darmouth Publishing Company.

Clark, T. N.; Hoffmann-Martinot, V. (eds.) (1998). The New Political Culture. Colorado: Westview Press.

Commaille, J. (1993). Les stratégies des femmes. Travail, famille et politique. París: La Découverte.

Commaille, J.; De Singly, F. (1997). «L'avenir politique de la question familiale en Europe». En: La Question Familiale en Europe. París: L’Harmattan, p. 307- 329.

COMISIÓN DE LAS COMUNIDADES EUROPEAS (2000). Hacia una estrategia marco comunitaria sobre la igualdad entre hombres y mujeres (2001-2005). Bruselas.

COMMISSION OF THE EUROPEAN COMMUNITIES (1991). Family and Employment within the Twelve. Bruselas: Directorate General for Employment, Industrial Relations and Social Affaires.

DALY, M.; LEWIS, J. (1998). «Conceptualising Social Care in the Context of Welfare State Restructuring». En: LEWIS, J. Gender, Social Care and Welfare State Restructuring in Europe. Ashgate, p. 1-24.

DalY, M. (1998). "A More Caring State? The Implications of Welfare State Restructuring for Social Care in the Republic of Ireland». En: LEWIS, J. Gender, Social care and Welfare State Restructuring in Europe. Ashgate, p. 28-50.

De Singly, F.; Comaille, J. (1997). «Les règles de la méthode comparative dans le domaine de la famille. Le sens d'une comparaison». En: La Question Familiale en Europe. París: L'Harmattan, p. 7-33.

DIENEL, C. (1995). "The institutionalisation and effectiveness of family policy in Europe». En: HANTRAIS, L.; LETABRIER, M.-T. The family in social policy and family policy in Europe. Leicestershire: The Cross-National Research Group. European Research Centre, p. 33-43. 
Dumon, W. (1990). Les politiques familiales des états membres de la communauté. Commission des Communautés Européennes. Direction Generale Emploi, Relations Industrielles et Affaires Sociales.

- (1997). "Les incertitudes des politiques à l'égard de la famille». En: COMAILle, J.; De Singly, F. La question familiale en Europe. París: L'Harmattan, p. $81-105$.

ESPING-ANDERSEN, G. (1999a). Social Foundations of Post-industrial Economies. Oxford: Oxford University Press.

- (1999b). «Épilogue pour l'édition française. Les trois mondes revus». En: Les trois mondes de l'État-providence. Essai sur le capitalisme moderne. París: Presses Universitaires de France.

- (2002). Why We Need a New Welfare State. Oxford: Oxford University Press.

European Commission (2002). Family Benefits and Family Policies in Europe.

Directorate-General for Employment and Social Affaires. Unit E.2.

EUROSTAT (2002). Feasibility study on the availability of comparable child care statistics in the European Union. Luxemburgo: European Commission. Directorate E- Unit E-2: Living conditions.

Ferrera, M. (1993). Modeli di Solidarietà. Politica e riforme sociali nelle democrazie. Bolonia: Il Mulino.

Flaquer, L. (ed.) (2002). Políticas familiares en la Unión Europea. Barcelona: Institut de Ciències Politiques i Socials, p. 9-27.

FLAQUER, L. (1995). "El modelo de familia española en el contexto europeo». En: SARASA, S.; MORENO, L. (eds.). El estado del bienestar en la Europa del sur. Madrid: Consejo Superior de Investigaciones Científicas e Instituto de Estudios Sociales Avanzados, p. 289-231.

- (2000a). Las políticas familiares en una perspectiva comparada. Valencia: Fundación La Caixa.

- (2000b). Family Policy and Welfare State in Southern Europe. Barcelona: Institut de Ciències Polítiques i Socials.

- (2004). «La articulación entre familia y estado del bienestar en los países de la Europa del sur». Papers, no 73: 27-58.

Forssén, K.; HAKOVIRTA. M. (2000). Family policy, work incentives and employment of mothers: Findings from de Luxembourg Income Study. Social Security in the Global Village. Helsinki: International Social Security Association (ISSA).

Gauthier, A. H. (1996a). The State and the Family. A Comparative Analysis of Family Policies in Industrialized Countries. Oxford: Clarendon Press.

- (1996b). "The Measured and Unmeasured Effects of Welfare Benefits on Families: Implications for Europe's Demographic Trends». En: COLEMAN, D. (ed.). Europe's Population in the 1990s. Oxford: Oxford University Press, p. 297-333.

- (1999). "The Sources and Methods of Comparative Family Policy Research». Comparative Social Research, 18: 31-56.

Gornick, J.; Meyers, M.; Ross, K. (1996). Public-Policies and the Employment of Mothers: A Cross-National Study. Luxembourg Income Study.

- (1997). «Supporting the Employment of Mothers: Policy Variation Across Fourteen Welfare States». Journal of European Social Policy, 7 (1): 45-70.

Hantrais, L.; Letabrier, M.-T. (1995). The Family in Social Policy and Family Policy in Europe. Leicestershire: The Cross-National Research Group. European Research Centre.

- (1996). Families and Family Policies in Europe. Nueva York: Longman. 
Hilamo, H. (2002). Family Policy Models and Family Policy Outcomes. A Nordic Perspective. Luxembourg Income Study.

KAMERMAN, S. (1990). «Parental Leave and Infant Care: U.S. and International Trends and Issues, 1978-1988». En: HyDE, J. S.; EsseX, M. J. Parental Leave and Child Care. Setting a Research and Policy Agenda. Filadelfia: Temple University Press, p. 11-24.

KenjoH, E. (2003). Women's Employment around Birth of the First Child in Britain, Germany, The Netherlands, Sweden and Japan. Essex: Institute for Social and Economic Research (ISER).

LaND, H.; LEWIS, J. (1998). "Gender, Care and the Changing Role of the State in the UK». En: LEWIS, J. Gender, Social Care and Welfare State Reconstructing in Europe. Ashgate, p. 51-84.

LEWIS, J. (1992). "Gender and the development of Welfare Regimes». Journal of European Social Policy, 2 (3): 159-173.

- (1997). "Gender and Welfare Regimes: Further Thoughts». Social Politics: 160177.

Mandel, H.; Semyonov, M. (2003). Welfare Family Policies and Gender Earnings Inequality: A Cross-National Comparative Analysis. Luxembourg Income Study.

Meyers, M.; Gornick, J.; Ross, K. (1999). «Public Childcare, Parental Leave, and Employment». En: SAInsburY, D. Gender and Welfare State Regimes. Oxford: Oxford University Press, p. 117-147.

MONTANARI, I. (2000). «From family wage to marriage subsidy and child benefits: controversy and consensus in the development of family support». Journal of European Social Policy, 10(4): 307-333.

Morgan, K. (2002). «Forging the Frontiers between State, Church and Family: Religious Cleavages and the Origins of Early Childhood Education and Care Policies in France, Sweden, and Germany». Politics and Society, 30. n. ${ }^{\circ} 1$ : 113-148.

NaLdini, M. (2003). The family in the Mediterranean Welfare States. Londres: Frank Cass.

Pitrou, A. (1995). «Some initial thoughts on cross-national comparisons of family policy measures: beyond words and declarations of intent». En: HANTRAIS, L.; Letabrier, M.-T. The family in social policy and family policy in Europe. Leicestershire: The Cross-National Research Group. European Research Centre, p. $10-15$.

RodríGUEZ, M. J. (2005). Familia y estado del bienestar: Estrategias de intervención y estrategias familiares en la Unión Europea (1970-1990). Tesis doctoral. Universidad Pablo de Olavide.

SAINSBURY, D. (1999a). «Taxation, Family Responsibilities and Employment». En: Gender and Welfare State Regimes. Oxford: Oxford University Press, p. 185-245.

- (1999c) «Gender, Policy Regimes, and Politics». En: Gender and Welfare State Regimes. Oxford: Oxford University Press, p. 244-277.

SARRACENO, Ch.; NALDINI, M. (2001). Sociología della famiglia. Bolonia: Il Mulino.

SCHEIWE, K. (1997). New demands for social protection-changing family structures, women's roles and institutional responses. The case of the German Long-Term Care Insurance. Mannheim: Manheim Centre for European Social Research.

SCHMidT, M. (1993). "Gendered Labour Force Participation». En: CASTLES, F. Families of Nations: Patterns of Public Policy in Western Democracies. Darmouth Publishing Company, p. 179-239. 
Semyonov, M. (1980). «The Social Context of Women's Labor Force Participation: A comparative Analysis». The American Journal of Sociology, 86(3): 534-550.

Simonen, L.; Kovalainen, A. (1998). «Paradoxes of Social Care Restructuring: The Finish Case». En: LeWIS, Jane (ed.). Gender, Social Care and Welfare State Restructuring in Europe. Aldershot, UK: Ashgate, p. 229-256.

WILENSKY, H. y otros (1987). "Comparative social policy: theories, methods, findings». En: Dierkes, M.; WeIler, H. N.; ANTAL, A. B. Comparative Policy Research. Learning from Experience. Hampshire: Gower, p. 381-458.

Zimmerman, S. (1995). Understanding Family Policy. Londres: Sage Publications. 